\title{
Direct Oral Anticoagulant Dosing in Extremes of Body Weight: Time to Revisit the Guidelines?
}

\author{
Arjun K. Pandey ${ }^{1}$ John W. Eikelboom $2,3,4$ \\ ${ }^{1}$ Michael G. DeGroote School of Medicine, McMaster University, \\ Hamilton, Ontario, Canada \\ 2 Department of Medicine, McMaster University, Hamilton, Ontario, \\ Canada \\ ${ }^{3}$ Thrombosis and Atherosclerosis Research Institute, Hamilton, \\ Ontario, Canada \\ ${ }^{4}$ Population Health Research Institute, Hamilton, Ontario, Canada
}

Thromb Haemost 2021;121:118-120.

Patients with high body weight are at increased risk of developing atrial fibrillation (AF) or venous thromboembolism (VTE) and are often treated with antithrombotic drugs. ${ }^{1}$ Direct oral anticoagulants (DOACs) have replaced vitamin K antagonists for many indications, but clinicians avoid DOACs in patients with high body weight because of concerns that decreased drug exposure will reduce efficacy. The International Society on Thrombosis and Haemostasis suggests avoiding DOACs in patients with body weight $\geq 120 \mathrm{~kg}$ or body mass index $(\mathrm{BMI})>40 \mathrm{~kg} / \mathrm{m}^{2}$, or routinely measuring drug-specific levels and continuing treatment only if the level is within the expected range. ${ }^{2}$

Two papers in this edition of the journal provide further insights into the potential use of DOACs in the long-term treatment of patients with extreme body weights. In the first study, Martin and colleagues measured DOAC drug levels in 100 patients with body weight $\geq 120 \mathrm{~kg}$ who were treated with standard therapeutic doses of apixaban ( $5 \mathrm{mg}$ twice daily) or rivaroxaban ( $20 \mathrm{mg}$ once daily) for stroke prevention in AF or for the management of VTE. ${ }^{3}$ Drug levels were determined by a chromogenic anti-Xa assay and compared with expected reference levels as reported in the International Council for Standardization in Hematology 2018 Consensus Document. ${ }^{4}$ The participant median age was 58 years, median body weight was $139 \mathrm{~kg}$ (minimum [min] 120, maximum [max] 230), and median BMI was $45 \mathrm{~kg} / \mathrm{m}^{2}$ (min 32, max 72). In participants treated with apixaban, 16 of 18 trough levels (89\%) and all 19 peak levels were within or above the expected range. In participants treated with rivaroxaban, all 21 trough levels and 32 of 58 peak levels (55\%) were within or above the expected range. There was no significant relationship between body weight, BMI, renal function, and drug levels.

In the second paper, Boriani and colleagues from the Thrombolysis In Myocardial Infarction (TIMI) group examined
Address for correspondence John W. Eikelboom, MBBS, Population Health Research Institute, 237 Barton Street East, Hamilton, ON L8L $2 \times 2$, Canada (e-mail: eikelbj@mcmaster.ca).

pharmacokinetic, pharmacodynamic, and clinical outcomes according to body weight in 4,328 patients enrolled in the AF-TIMI-48 trial who were randomized to one of two edoxaban dosing regimens (higher dosing regimen: $60 \mathrm{mg}$ once daily, lower dosing regimen: $30 \mathrm{mg}$ once daily; for both regimens the dose was halved if the estimated creatinine clearance was $<50 \mathrm{~mL} / \mathrm{min}$, weight $<60 \mathrm{~kg}$, or there was concomitant use of a potent P-glycoprotein inhibitor) or warfarin. ${ }^{5}$ Drug levels were measured by a chromogenic anti-Xa assay and were compared in patients with high (95th-100th percentile, $n=1,093$ ), middle (45th-55th percentile, $n=2,153)$, and low ( $0-5$ th percentile, $n=1,082$ ) body weights. Median ages by weight category were 62,73 , and 76 years, respectively; median body weights were $130 \mathrm{~kg}(\min 120, \max 227), 82 \mathrm{~kg}(\min 80, \max 84)$, and $51 \mathrm{~kg}(\min 30, \max 55)$, respectively. Trough and peak levels for both the higher and lower edoxaban dosing regimens were not significantly different across weight categories ${ }^{5}$ and, although not evaluated by the authors, appeared to be comparable to the expected levels. ${ }^{3}$ Patients with high body weights had lower rates of stroke or systemic embolism and major bleeding than those with middle or low body weights. The efficacy of edoxaban compared with warfarin for stroke prevention was consistent across body weight categories, but edoxaban produced a greater reduction in bleeding in patients with low body weights, and this translated into a larger net benefit (composite of stroke, systemic embolism, major bleeding, and death) for these patients. Separate analyses comparing patients with weight $>150 \mathrm{~kg}(n=84)$ to those with weight 120 to $150 \mathrm{~kg}$ $(n=474)$ revealed similar median trough levels but lower peak levels ( $p$-values not presented for these comparisons).

The pharmacokinetic results reported by both Martin et al ${ }^{3}$ and Boriani et $\mathrm{al}^{5}$ confirm preliminary data from previous studies $^{6-8}$ that almost all patients with body weight $>120 \mathrm{~kg}$ will achieve expected trough drug levels with the use of received

August 16, 2020

accepted

August 16, 2020

published online

September 17, 2020 (c) 2020. Thieme. All rights reserved. Georg Thieme Verlag KG,

Rüdigerstraße 14,

70469 Stuttgart, Germany
DOI https://doi.org/

10.1055/s-0040-1716753. ISSN 0340-6245. 


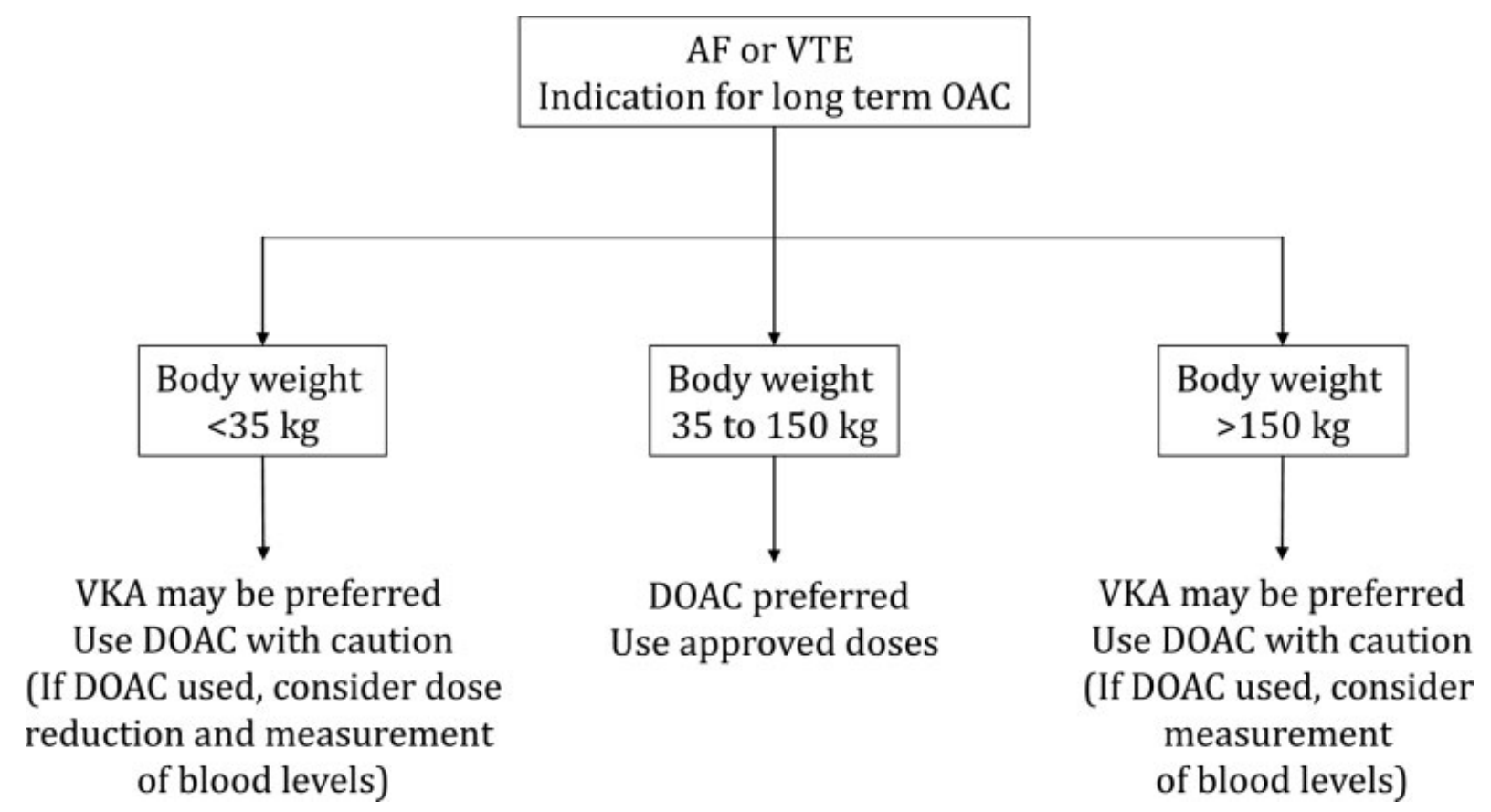

Fig. 1 Proposed use of direct oral anticoagulants in patients with body weight $<35,35-150$, and $>150 \mathrm{~kg}$. AF, atrial fibrillation; DOAC, direct oral anticoagulant; OAC, oral anticoagulation; VKA, vitamin K antagonist; VTE, venous thromboembolism.

apixaban, edoxaban, and rivaroxaban. Most of these patients will also achieve expected peak levels with apixaban but some achieve lower than expected peak levels with the once-daily DOACs, edoxaban, and rivaroxaban. However, lower than expected peak levels did not appear to compromise efficacy; Boriani et al demonstrated consistent benefits with edoxaban compared with warfarin in patients with body weight $>120 \mathrm{~kg} .{ }^{5}$ Similarly, consistent benefits of DOACs have been reported from the ARISTOTLE trial comparing apixaban with warfarin for stroke prevention in patients with AF and body weight $>120 \mathrm{~kg}^{9}{ }^{9}$ as well as in a meta-analysis of AF trials examining outcomes with DOACs compared with warfarin according to BMI. ${ }^{10}$

Despite the overall reassuring results from the analyses by Martin et al and Boriani et al with the use of DOACs in patients with high body weights, several caveats should be considered. First, drug levels are poorly validated as a surrogate for clinical outcomes. Although it is possible to accurately measure DOAC drug levels, patient characteristics (e.g., age, renal function) confound the association with clinical outcome, a single measurement of drug levels is unreliable, and optimal drugs remain to be established. ${ }^{11}$ Second, event rates for both thromboembolism and bleeding are low in patients with high body weights, and this limits the power to detect clinically important treatment interactions. Third, despite representing two of the larger studies on this topic, the reports by Martin et al and Boriani et al collectively included very few patients with body weight $>150 \mathrm{~kg}$ and clinical outcome data for this extreme weight group remain limited.

What are the implications of these findings for clinical practice? We believe that there is an urgent need for revision of the guidelines concerning the use of DOACs in patients with high body weights. We suggest that updated guidelines should recommend DOACs in preference to warfarin for patients with
AF or VTE and body weights up to $150 \mathrm{~kg}$ without routine measurement of drug levels (- Fig. 1). Whether DOACs should also be preferred in patients with body weights above $150 \mathrm{~kg}$ remains an open question, but we suspect that even in these patients the greater convenience of DOACs will make them an attractive option. At the other end of the weight spectrum, DOACs should likely also be preferred because patients with low body weight not only are at higher risk of thromboembolism than patients with middle or high body weights, but also appear to derive greater benefits of treatment with a DOAC. At the same time, body weights should not be considered in isolation, but should be incorporated as part of an integrated approach to patient care. ${ }^{12}$ In patients with $\mathrm{AF}$ and extremes of body weight, recommendations regarding antithrombotic management should also take into account other clinical risk factors for thromboembolism and bleeding using the $\mathrm{CHA}_{2} \mathrm{DS}_{2}$ VASc score ${ }^{13}$ and possibly biomarkers, ${ }^{14}$ as well as changing risk over time. ${ }^{15}$

What are the implications for future research? Despite the advances to date, we echo Boriani and colleagues in calling for additional randomized outcome data in patients at the greatest extremes of body weight ( $<35$ or $>150 \mathrm{~kg}$ ). We encourage ongoing and future trials of DOACs to actively enroll patients with extremes of body weights, and to consider routine measurement of steady-state trough and peak levels in at least a subset of these patients. In the meantime, additional information on the efficacy and safety of DOACs compared with warfarin in AF in patients at extremes of body weight is expected from an ongoing collaborative meta-analysis of the phase 3 randomized controlled trials in patients with AF.

Conflict of Interest

J.W.E. reports honoraria and grant support from Astra Zeneca, Bayer, Boehringer Ingelheim, Bristol-MyersSquibb/Pfizer, Daiichi Sankyo, Glaxo Smith Kline, Janssen, 
sanofi aventis and Eli Lilly as well as a personnel award from the Heart and Stroke Foundation.

\section{References}

1 Afshin A, Forouzanfar MH, Reitsma MBGBD 2015 Obesity Collaborators, et al. Health effects of overweight and obesity in 195 countries over 25 years. N Engl J Med 2017;377(01):13-27

2 Martin K, Beyer-Westendorf J, Davidson BL, Huisman MV, Sandset PM, Moll S. Use of the direct oral anticoagulants in obese patients: guidance from the SSC of the ISTH. J Thromb Haemost 2016;14 (06):1308-1313

3 Martin A-C, Thomas W, Mahir Z, et al. Direct oral anticoagulant concentrations in obese and high body weight patients: a cohort study. Thromb Haemost 2020;120(02):224-233

4 Gosselin RC, Adcock DM, Bates SM, et al. International Council for Standardization in Haematology (ICSH) recommendations for laboratory measurement of direct oral anticoagulants. Thromb Haemost 2018;118(03):437-450

5 Boriani G, RuffC, Kuder JF, et al. Edoxaban versus warfarin in patients with atrial fibrillation at the extremes of body weight: an analysis from the ENGAGE AF-TIMI 48 trial. Thromb Haemost 2020;121(02): 140-149

6 Piran S, Traquair H, Chan N, Bhagirath V, Schulman S. Peak plasma concentration of direct oral anticoagulants in obese patients weighing over 120 kilograms: a retrospective study. Res Pract Thromb Haemost 2018;2(04):684-688

7 Wasan SM, Feland N, Grant R, Aston CE. Validation of apixaban anti-factor Xa assay and impact of body weight. Thromb Res 2019; 182:51-55

8 Arachchillage D, Reynolds R, Devey T, Maclean R, Kitchen S, van Veen JJ. Effect of extremes of body weight on drug level in patient treated with standard dose of rivaroxaban for venous thromboembolism; real life experience. Thromb Res 2016; 147:32-35

9 Hohnloser SH, Fudim M, Alexander JH, et al. Efficacy and safety of apixaban versus warfarin in patients with atrial fibrillation and extremes in body weight. Circulation 2019;139(20): 2292-2300

10 Wang SY, Giugliano RP. Non-vitamin K antagonist oral anticoagulant for atrial fibrillation in obese patients. Am J Cardiol 2020; 127:176-183

11 Eikelboom JW, Quinlan DJ, Hirsh J, Connolly SJ, Weitz JI. Laboratory monitoring of non-vitamin $\mathrm{K}$ antagonist oral anticoagulant use in patients with atrial fibrillation: a review. JAMA Cardiol 2017;2(05):566-574

12 Yoon M, Yang PS, Jang E, et al. Improved population-based clinical outcomes of patients with atrial fibrillation by compliance with the simple $A B C$ (Atrial Fibrillation Better Care) pathway for integrated care management: a nationwide cohort study. Thromb Haemost 2019;119(10):1695-1703

13 de Vries TAC, Hirsh J, Xu K, et al. Apixaban for stroke prevention in atrial fibrillation: why are event rates higher in clinical practice than in randomized trials?-A systematic review. Thromb Haemost 2020;120(09):1323-1329

14 Esteve-Pastor MA, Roldán V, Rivera-Caravaca JM, Ramírez-Macías I, Lip GYH, Marín F. The use of biomarkers in clinical management guidelines: a critical appraisal. Thromb Haemost 2019;119(12): 1901-1919

15 Chao TF, Liao JN, Tuan TC, et al. Incident co-morbidities in patients with atrial fibrillation initially with a CHA2DS2-VASc score of 0 (males) or 1 (females): implications for reassessment of stroke risk in initially 'low-risk' patients. Thromb Haemost 2019;119 (07):1162-1170 